\title{
A Movable Thomson Scattering System Based on Optical Fibers (TS-Probe)
}

\author{
Kazumichi NARIHARA and Hiroshi HAYASHI \\ National Institute for Fusion Science, 322-6 Oroshi-cho, Toki 509-5292, Japan
}

(Received 9 January 2009 / Accepted 8 July 2009)

\begin{abstract}
This paper proposes a movable compact Thomson scattering (TS) system based on optical fibers (TS-probe). The TS-probe consists of a probe head, optical fibers, a laser diode, a polychromator, and lock-in amplifiers. Laser beam optics and light collection optics are mounted rigidly on a probe head with a fixed scattering position. Laser light and scattered light are transmitted by flexible optical fibers, enabling us to move the TS-probe head freely during plasma discharge. The light signal scattered from an amplitude-modulated laser is detected against the plasma light based on the principle of the lock-in amplifier. With a modulated laser power of $300 \mathrm{~W}$, the scattered signal from a sheet plasma $15 \mathrm{~mm}$ deep with $n_{\mathrm{e}} \sim 10^{19} \mathrm{~m}^{-3}$ can be measured with $10 \%$ accuracy by setting the integrating time to $0.1 \mathrm{~s}$. The TS-probe head is similar to a $1 / 20$ model of the currently operating Large Helical Device-TS.
\end{abstract}

(C) 2010 The Japan Society of Plasma Science and Nuclear Fusion Research

Keywords: Thomson scattering system, fiber laser, divertor plasma, TS-probe, LHD

DOI: $10.1585 /$ pfr.5.S1046

\section{Introduction}

In fusion-oriented plasma experiments, Thomson scattering (TS) is a standard method of obtaining electron temperature $\left(T_{\mathrm{e}}\right)$ and density $\left(n_{\mathrm{e}}\right)$ profile data. Its advantage is that it gives local values of $T_{\mathrm{e}}$ and $n_{\mathrm{e}}$ with high confidence and accuracy. There are, however, disadvantages; the small probability of TS $\left(n_{\mathrm{e}} \sigma L<10^{-11}\right)$ necessitates bright optics and highly efficient detectors even with currently available high-energy pulse lasers, and precise laser beam alignment is often required. As a result, TS typically is costly and requires a tedious labor in construction and maintenance, preventing its easy use at various locations in a plasma confinement device. As a possible solution, this paper proposes the concept of a TS-probe that is compact and can be moved, like a Langmuir probe, to a desired position around a plasma without requiring laser beam alignment. We proposed a similar idea 13 years ago [1], but it has not yet been realized because a suitable light source had not been available. Recently developed laser diodes that can deliver a power as high as $300 \mathrm{~W}$ at $980 \mathrm{~nm}$ seem to be attractive for this scheme and motivate us to re-examine the idea in this paper.

\section{Description of a TS-Probe}

We envision measuring a divertor-leg plasma of 15 $\mathrm{mm}$ depth with a TS-probe as shown in Fig. 1.

It consists of a probe head, optical fibers, optical fiber feed-through, a laser diode, a polychromator, and lock-in amplifiers. These are described in details below.

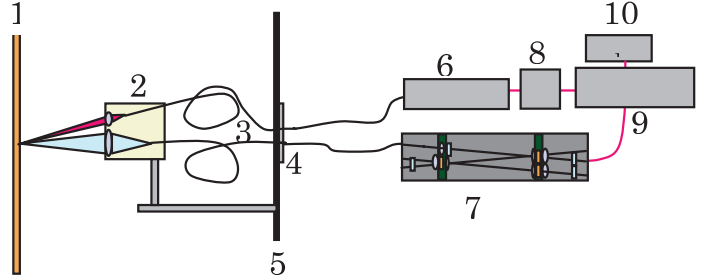

Fig. 1 Schematic view of a TS-probe: 1. divertor-leg plasma; 2. TS-probe head; 3. optical fibers; 4. fiber feed-through; 5. vacuum chamber wall; 6. laser diode; 7. polychromator; 8. pulse generator; 9. lock-in amplifier; 10. data acquisition.

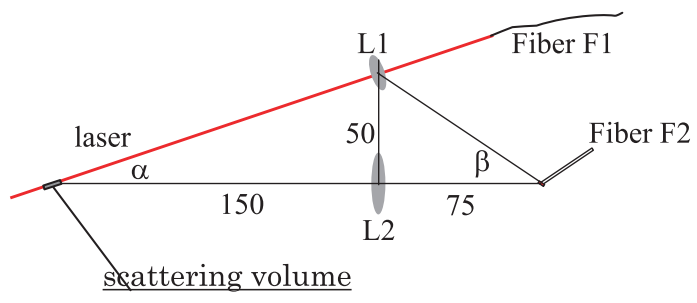

Fig. 2 Thomson scattering optics configuration. L1 and L2 are lenses.

(1) Probe Head: This is a compact optical bench on which laser delivery and light collection optics are firmly mounted. A key point is to collect as many TS photons as possible while keeping background plasma light at the smallest level. For this, it is important to form an image of the laser beam just on the surface of the light collection fiber with a slit of well-adjusted size. Figure 2 shows 
the scattering configuration. In a simple geometry, the image of the oblique line (laser beam), which intersects L2's optical axis at an angle $\alpha$, intersects the optical axis at $\beta$, which is related to $\alpha$ by $\tan \alpha / \tan \beta=m$. Here, $m=75 / 150=1 / 2$ is the magnification.

An expanded view of the end of the optical fiber is shown in Fig. 3. The incident angle $\theta_{1}=90^{\circ}-\beta$ is chosen so that light incident to the fiber is reflected as little as possible. If the laser beam, and hence the scattered light are properly polarized ( $\mathrm{p}$-waves), the reflectance at the fiber surface is less than $1 \%$ for $45^{\circ}<\theta_{1}<63^{\circ}$ as shown in Fig. 4. Unfortunately, the fiber we planned on using depolarizes the propagating laser light even if it is initially polarized. The unpolarized light reflects from the surface of the fiber by $5 \%$ on average for $45^{\circ}<\theta_{1}<63^{\circ}$, as shown in Fig. 4. We choose a central incident angle of $\theta_{1}=56^{\circ}$; hence, $\beta=34^{\circ}$ and $\alpha=17^{\circ}$ for $m=0.5$. The end of the fiber is cut at an angle of $56^{\circ}$ so that centrally incident light propagates along the axis of the fiber after refraction. A slit $1 \mathrm{~mm}$ in length and $0.5 \mathrm{~mm}$ in width set at the obliquely cut surface of the fiber defines the scattering cylindrical volume $L=3 \mathrm{~mm}$ in length and $D=1 \mathrm{~mm}$ in diameter located at the waist point of the laser beam shaped by lens

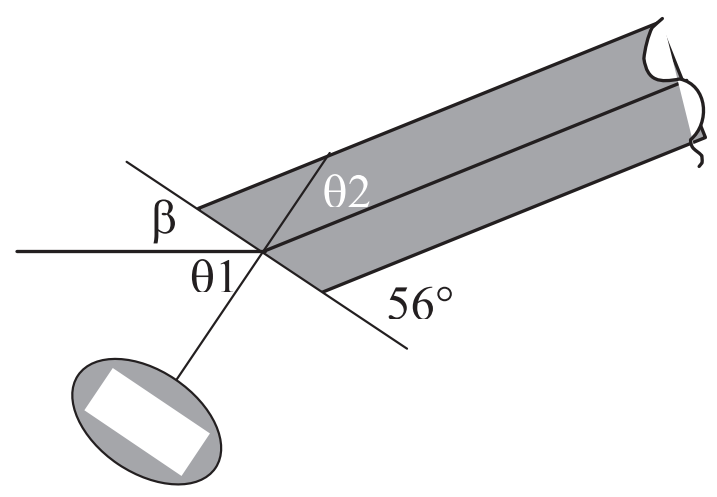

Fig. 3 Magnified view of the optical fiber for collecting TS light.

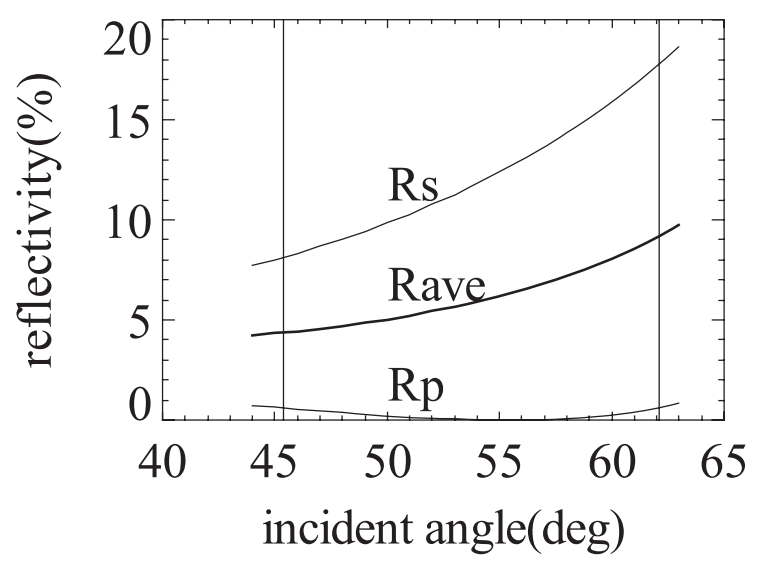

Fig. 4 Reflectivity at the end surface of the fiber as a function of incident angle.
L1. L1 has a focal length $f=34 \mathrm{~mm}$ and diameter $\phi 30$, which matches $N A=0.22$ of fiber $\mathrm{F} 1$ with a core diameter of $0.5 \mathrm{~mm}$.

Lens L2 has a focal length $f=52 \mathrm{~mm}$ and is $40 \mathrm{~mm}$ in diameter with a $34 \mathrm{~mm} \times 40 \mathrm{~mm}$ square aperture, which matches the acceptance angle of fiber F2. The aperture gives a solid angle of $\Delta \Omega=25 \mathrm{msr}$. All optical components making up the TS probe are housed in a box $100 \times 100 \times 50 \mathrm{~mm}^{3}$ in size. The scattering configuration is similar to a $1 / 20$ model of the Large Helical Device-TS (LHD-TS).

(2) Optical Fibers: Two optical fibers are used. F1 delivers high-power laser energy, and F2 transports the scattered light to a polychromator. Contrary to our intuition, it is reported that a step-index type optical fiber made of pure silica can transmit a high power density [2]; a $400 \mu \mathrm{m}$ core diameter fiber can transmit $2000 \mathrm{~W}$ continuous wave (CW) light and a $1 \mathrm{MW} / \mathrm{mm}^{2}$ light pulse width of $<1 \mathrm{~ms}$. We found no progress in the transmittable power density in fibers in the past 13 years, probably indicating a mature state of the art. Considering an available laser diode of $300 \mathrm{~W} \mathrm{CW}$ output, a core diameter of $500 \mu \mathrm{m}$ is large enough for fiber F1. Lens L2 $\left(f=52 \mathrm{~mm} ; 20 \times 30 \mathrm{~mm}^{2}\right.$ aperture) focuses the scattered light onto the end surface of $\mathrm{F} 2$ at a 1-mm diameter cut at the Brewster angle.

Both fibers, set inside a vacuum chamber, are flexible enough that we can move the TS-probe head freely to a large extent. A simple metallic screen shields the fibers against plasma radiation that would otherwise heat them. Unavoidable exposure to hard x-rays and neutrons, if present, will result in a gradual drop in the transmission of the fibers and fluorescent emission from the body of the fibers. The latter problem will not be serious for the present case, in which a modulated laser beam is used. The former problem will be alleviated greatly by selecting a suitable fiber material and wavelength for the laser light (900-1100 nm) transmitted in the fiber. An increase in the temperature of the fibers, which may occur naturally or intentionally, will remove the defects introduced inside them.

(3) Fiber Feed-Through: This new product is useful for interfacing the fibers set inside and outside of a vacuum chamber. A commercially available fiber feed-through can be used for an ultra-high vacuum (UHV) environment of $10^{-10}$ mbar [3]. Durability against high power transmission must be investigated.

(4) Light Sources: The light source for TS must be intense enough to yield a sufficient signal and have a wavelength width narrow enough to discern Doppler broadening of the scattered spectrum due to thermal motion of plasma electrons. The allowable width $(\Delta \lambda)$ depends on the minimummeasurable electron temperature $T_{\mathrm{e}_{-} \min }(\mathrm{eV})$, as follows: $\Delta \lambda / \lambda \ll T_{\text {e_min }}^{0.5} / 500$. For example, for $T_{\text {e_min }}=10 \mathrm{eV}$, 
$\Delta \lambda / \lambda \ll 6 / 1000$; and for $T_{\text {e_min }}=1 \mathrm{eV}, \Delta \lambda / \lambda \ll 2 / 1000$. These values imply that it is not absolutely necessary to use lasers operating in a cavity, e.g., an Nd:YAG laser with $\Delta \lambda / \lambda \ll 10^{-6}$, as a TS light source. Laser diodes, which are widely used for pumping the laser rod and for industrial applications such as welding and cutting, seem suitable for the present purpose. Great progress in the power and beam quality of laser diodes has been made in the past decade. For example, LIMO GmbH produces a fiber-coupled laser diode module that delivers $980 \mathrm{~nm}, 350 \mathrm{~W}$ light from a $400 \mu \mathrm{m}$ core fiber with $N A=0.22$ [4]. The specified line width (FWHM) $\Delta \lambda<8 \mathrm{~nm}$ will allow $T_{\mathrm{e}}$ measurement to come down to $T_{\text {e_min }} \sim 30 \mathrm{eV}$, which is rather high for edge plasma measurement. Recently it was reported [5] that a Bragg grating stabilizes a diode laser to yield a power of $330 \mathrm{~W}$ from a fiber with $\Delta \lambda=0.5 \mathrm{~nm}$ line width at $\lambda=967 \mathrm{~nm}$, allowing TS measurement down to a few eV. The above data are for $\mathrm{CW}$ operation. As described later, the laser diode should be modulated at a high frequency. Whether the ability to deliver high power degrades when the laser diode is used in a modulation mode is an open question.

(5) Polychromator: We will use a conventional three-filter polychromator. The principle of operation is based on the transmission and reflection characteristics of a combination of filters arranged to form a zigzag optical path. A key concern is to prevent stray light with the wavelength of the laser from entering the light detector, such as an avalanche photo diode (APD). In the case of the LHD-TS, each filter in the polychromator blocks the light at $1064 \mathrm{~nm}$ (the Nd: YAG laser wavelength) with a blocking factor of $10^{5}$ (OD5). Even with this high blocking factor, some polychromators suffer from stray light. Considering that the power is as high as $300 \mathrm{~W}$ in the present case, the filters with a much higher OD level will be necessary. Setting a notch filter with OD $\sim 4$ at the laser wavelength at the exit of the fiber-lens may be a solution. In addition to the strong blocking, the steepness of the edge of the filters is important determining the lowest detectable $T_{\mathrm{e}}\left(T_{\mathrm{e} \_ \text {min }}\right)$. Recently developed volume holographic optical filters [6] with narrow band-widths seem promising for solving this problem.

(6) Lock-in Amplifiers: These are used to pickup weak scattered signals modulated at frequency $f_{\mathrm{m}}$ in the presence of overwhelming background noise arising mainly from plasma light. In considering that the spectrum of plasma density fluctuation and the resultant plasma light fluctuation monotonically decreases down to the sub- $\mathrm{MHz}$ region, a reference frequency $f_{\mathrm{m}}$ higher than $1 \mathrm{MHz}$ is favorable for reducing the noise contribution. A lock-in amplifier that operates at frequencies as high as $200 \mathrm{MHz}$ is available: SR844 (Standard Research System) [7].

\section{Signal-to-Noise Ratio}

To assess the possible performance of the TS-probe described above, we estimate the accuracy of $T_{\mathrm{e}}$ and $n_{\mathrm{e}}$ values deduced from the outputs of the lock-in amplifiers. We consider a sheet-like divertor-leg plasma $15 \mathrm{~mm}$ deep with $T_{\mathrm{e}}$ less than $100 \mathrm{eV}$ and $n_{\mathrm{e}}$ ranging from $10^{18} \mathrm{~m}^{-3}$ to $10^{20} \mathrm{~m}^{-3}$ with a modulated laser diode of $P_{\text {LASER }}=300 \mathrm{~W}$ and $\lambda_{\text {LASER }}=980 \mathrm{~nm}$.

A backward TS spectrum is shown in Fig. 5 for various $T_{\mathrm{e}}$, which are discriminated by three color channels with pass-bands B1 $=[940,960], \mathrm{B} 2=[960,970]$ and B3 $=[970,975]$ in nm unit. The light power fed to a polychromator is the sum of the scattered light $P_{\text {sct }}$ plus the plasma radiation $P_{\text {rad }}$, which are given respectively as

$$
\begin{aligned}
& P_{\mathrm{sct}}=P_{\text {LASER }} n_{\mathrm{e}} L\left(d^{2} \sigma / \mathrm{d} \Omega \mathrm{d} \lambda\right) \Delta \Omega \Delta \lambda \\
& \approx 5.3 \times 10^{-19} P_{\mathrm{LASER}} n_{\mathrm{e}} L \Delta \Omega S p(\lambda) \mathrm{d} \lambda / \lambda_{\mathrm{LASER}}
\end{aligned}
$$

and

$$
\begin{aligned}
& P_{\mathrm{rad}}=j_{v} \Delta v \Delta V \Delta \Omega \\
& \approx 1.5 \times 10^{-45} Z_{\mathrm{eff}} n_{\mathrm{e}}^{2} T_{\mathrm{e}}^{-1 / 2} G \Delta \Omega V_{\mathrm{obs}} c \Delta \lambda / \lambda^{2} .
\end{aligned}
$$

Here, $S p(\lambda)$ is the TS spectrum shown in Fig. 5, $\lambda_{\text {LASER }}=$ $980 \mathrm{~nm}$ is the laser wavelength, $j_{v}$ is the bremsstrahlung emissivity, $Z_{\text {eff }}$ is the effective ion charge, $G$ is the Gaunt factor enhanced $\sim 5$ times to take account of enhanced radiation due to recombination and line radiation, $V_{\mathrm{obs}} \sim$ $\pi D^{2} / 4 \times 15 \sim 10 \mathrm{~mm}^{3}$ is the effective plasma volume that the collection optics see. The light power that falls in $i$ th filter's passband is incident to the $i$-th APD, inducing a current

$$
\begin{aligned}
& j_{i}=\int \mathrm{d} \lambda\left(P_{\mathrm{sct}}+P_{\mathrm{rad}}\right) F_{i}(\lambda) \lambda / h c \\
& \equiv j_{\mathrm{s} i}+j_{\mathrm{b} i} .
\end{aligned}
$$

Here, $F_{i}(\lambda)$ is the filter transmittance $\eta_{\mathrm{FIL}}$ times the quantum efficiency $\eta_{\mathrm{Q}}$ of the i-th channel: $F_{i}(\lambda)=\eta_{\mathrm{FIL}}(\lambda) \eta_{\mathrm{Q}}(\lambda)$.

We assume an idealized filter with $\eta_{\mathrm{FIL}}=0.9$ in the $i$-th passband and $\eta_{\mathrm{FIL}}=0$ otherwise. The responsivity

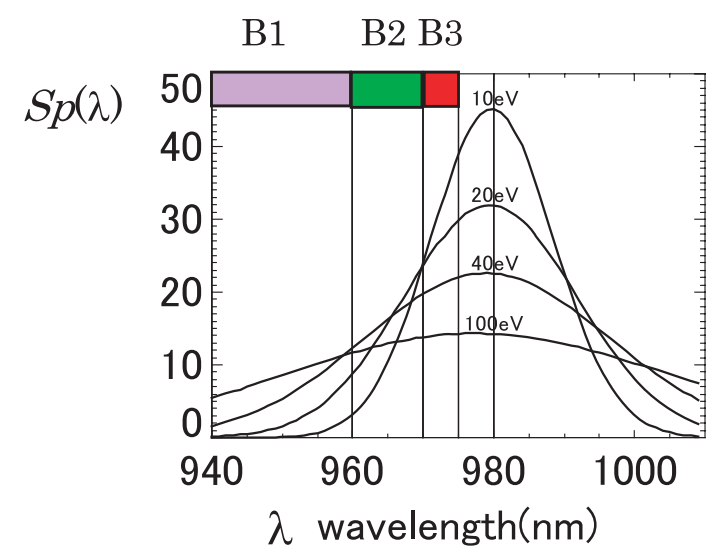

Fig. 5 TS spectra for various $T_{\mathrm{e}}$. Three color bands are indicated. 


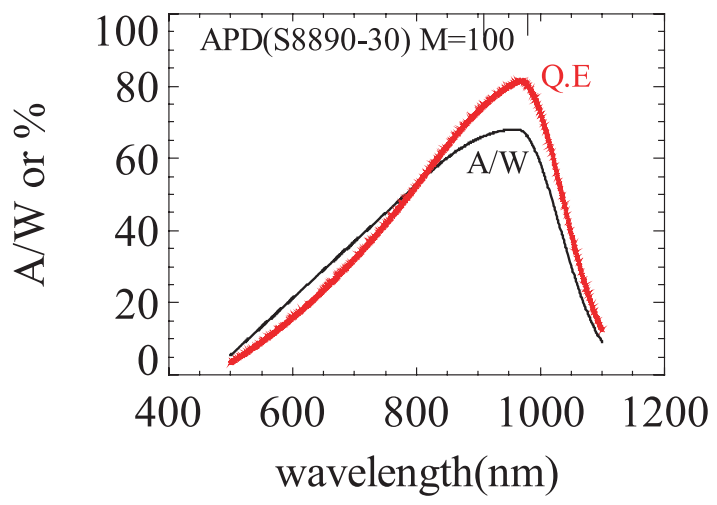

Fig. 6 Responsivity and quantum efficiency of an APD (Hamamatsu S8890).

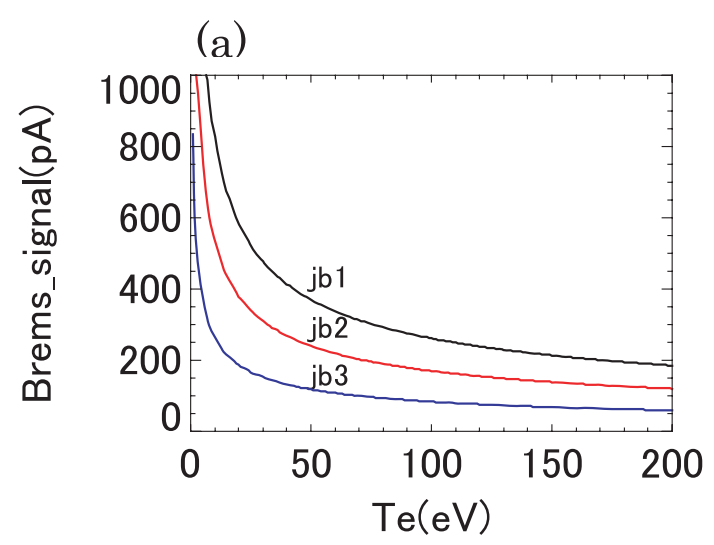

(b)

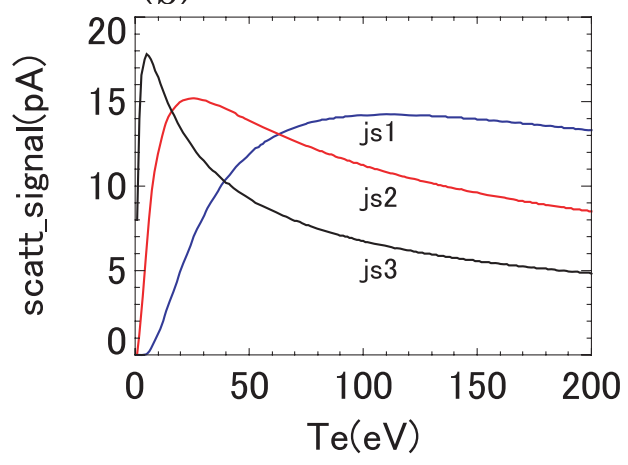

Fig. 7 APD currents induced by (a) bremsstrahlung and (b) Thomson scatted light. $n_{\mathrm{e}}=10^{19} \mathrm{~m}^{-3}$.

(A/W) and its associated quantum efficiency $\eta_{Q}(\%)$ of an APD are shown in Fig. 6 as functions of wavelength, from which we reasonably set $\eta_{\mathrm{Q}}=0.75$ for the three passbands $(940 \mathrm{~nm}<\lambda<975 \mathrm{~nm})$. Figures 7 (a) and (b) show $j_{\mathrm{b} i}$ and $j_{\mathrm{s} i}(i=1-3)$ for a plasma with $n_{\mathrm{e}}=10^{19} \mathrm{~m}^{-3}$ as a function of $T_{\mathrm{e}}$. We note that the scattering signals $j_{\mathrm{s} i} ; i=$ 1-3 are deeply buried in the plasma bremsstrahlung signals $\left\{j_{\mathrm{b} i} ; i=1-3\right\}$. The APD outputs, which are proportional to $j_{i}=j_{\mathrm{s} i}+j_{\mathrm{b} i}$ are fed to three lock-in amplifiers. A lock-in amplifier is a kind of Fourier analyzer if the reference in-

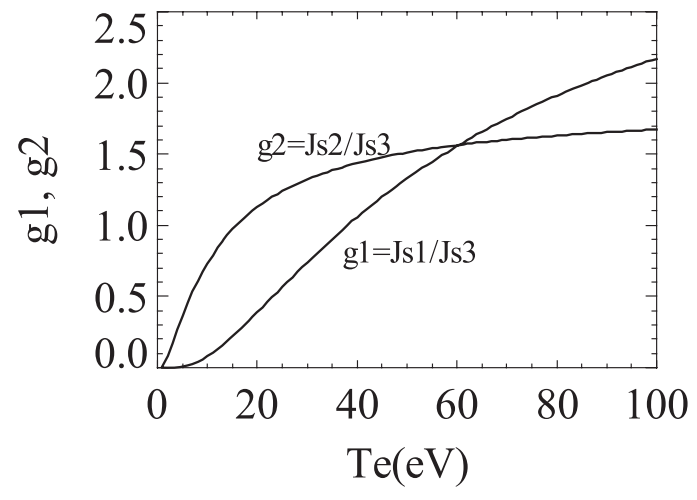

Fig. 8 Two ratios among the TS signals from three-color channels.

put is fed by a precise sinusoidal signal, which is also used to modulate the laser diode. The frequency spectrum of an APD's output is composed of two terms: a very narrow TS signal centered at the modulation frequency $f_{\mathrm{m}}$ and broadband noise generated mainly by shot noise. The current in the APD $j_{i}=j_{\mathrm{s} i}+j_{\mathrm{b} i}+j_{\mathrm{d}}$ generates the shot noise, whose power spectrum is given by

$$
<i_{\mathrm{n}}^{2}>=2 e j_{i} M^{2} F_{\mathrm{n}}(M) \Delta f_{\mathrm{APD}},
$$

where $e$ is the elementary charge, $M \sim 100$ is the multiplication factor of the APDs, $F_{\mathrm{n}}(M)$ is an excess noise factor, and $\Delta f_{\mathrm{APD}}$ is the bandwidth. Here, an inevitable dark current $j_{\mathrm{d}} \sim 1 \mathrm{nA}$ is added to the APD current. The appearance of $F_{\mathrm{n}}(M)$ implies enhanced noise in the avalanche process in the APD [8-10]. $F_{\mathrm{n}}(M)$ is dependent on the ratio of hole ionization to electron ionization. By suitable design and selection of the processing material, $F_{\mathrm{n}}(M)$ can be less than 4 for $M=100$ [10]. In this paper, we assume $F_{\mathrm{n}}(M)=10$. The RC-type integration time $\tau$ of a lock-in amplifier yields a spectrum width $\Delta f_{\mathrm{BW}}$ given by the relation $\Delta f_{\mathrm{BW}}=1 / 4 \tau$. Then, the output of the lock-in amplifier is expressed as

$$
y_{i}=j_{\mathrm{s} i} \pm\left(e j_{i} / 2 \tau\right)^{1 / 2} \equiv j_{\mathrm{s} i} \pm \delta y_{i} .
$$

Hence, however deeply the TS-light is buried in the plasma radiation, it is possible to pick up the TS signal by taking a long enough integration time.

With these data $\left\{y_{i} ; i=1-3\right\}$ we can estimate the errors in the deduced $T_{\mathrm{e}}$ and $n_{\mathrm{e}}$ by following the standard method. The two ratios $j_{\mathrm{s} 1} / j_{\mathrm{s} 3} \equiv g_{1}\left(T_{\mathrm{e}}\right)$ and $j_{\mathrm{s} 2} / j_{\mathrm{s} 3} \equiv g_{2}\left(T_{\mathrm{e}}\right)$ are functions of $T_{\mathrm{e}}$ alone, as shown in Fig. 8. Conversely, the observed ratios $y_{1} / y_{3}$ and $y_{2} / y_{3}$ give two $T_{\mathrm{e}}$ values: $y_{1} / y_{3}=$ $g_{1}\left(T_{\mathrm{e} 1}\right)$ and $y_{2} / y_{3}=g_{2}\left(T_{\mathrm{e} 2}\right)$. The errors in $T_{\mathrm{e} 1}$ and $T_{\mathrm{e} 2}$ are calculated as

$$
\begin{aligned}
& \left(\delta T_{\mathrm{e} 1}\right)^{2}=\left(g_{1}^{\prime}\right)^{-2}\left(\delta y_{1}^{2} / y_{3}^{2}+y_{1}^{2} \delta y_{3}^{2} / y_{3}^{4}\right), \\
& \left(\delta T_{\mathrm{e} 2}\right)^{2}=\left(g_{2}^{\prime}\right)^{-2}\left(\delta y_{2}^{2} / y_{3}^{2}+y_{2}^{2} \delta y_{3}^{2} / y_{3}^{4}\right), \\
& \left(\delta y_{i}\right)^{2}=e j_{i} / 2 \tau \quad(i=1-3) .
\end{aligned}
$$


With the variance $\left(\delta T_{\mathrm{e}}\right)^{2}$ defined by

$$
1 /\left(\delta T_{\mathrm{e}}\right)^{2}=1 /\left(\delta T_{\mathrm{e} 1}\right)^{2}+1 /\left(\delta T_{\mathrm{e} 2}\right)^{2},
$$

the most probable $T_{\mathrm{e}}$ is evaluated from the two ratios weighted by the reciprocal of the error in each ratio as

$$
T_{\mathrm{e}}=\left(T_{\mathrm{e} 1} /\left(\delta T_{\mathrm{e} 1}\right)^{2}+T_{\mathrm{e} 2} /\left(\delta T_{\mathrm{e} 2}\right)^{2}\right)\left(\delta T_{\mathrm{e}}\right)^{2} .
$$

The error in $n_{\mathrm{e}}$ is obtained, for example, from $y_{3}$ :

$$
\left(\delta n_{\mathrm{e}} / n_{\mathrm{e}}\right)^{2}=\left(\delta y_{3} / y_{3}\right)^{2}+\left(\delta j_{\mathrm{s} 3} / \partial T_{\mathrm{e}}\right)^{2}\left(\delta T_{\mathrm{e}}\right)^{2} / j_{\mathrm{s} 3}^{2} .
$$

Figure 9 shows the relative errors in $T_{\mathrm{e}}$ and $n_{\mathrm{e}}, \sqrt{\delta\left(T_{\mathrm{e}}\right)^{2}} / T_{\mathrm{e}}$ and $\sqrt{\left(\delta n_{\mathrm{e}}\right)^{2}} / n_{\mathrm{e}}$, as a function of $T_{\mathrm{e}}$ for four combinations of parameters:

(a) $\quad n_{\mathrm{e}}=10^{19} \mathrm{~m}^{-3}, P_{\text {LASER }}=300 \mathrm{~W}$ and $\tau=0.1 \mathrm{~s}$;

(b) $n_{\mathrm{e}}=10^{19} \mathrm{~m}^{-3}, P_{\text {LASER }}=100 \mathrm{~W}$ and $\tau=0.5 \mathrm{~s}$;

(c) $n_{\mathrm{e}}=10^{20} \mathrm{~m}^{-3}, P_{\text {LASER }}=300 \mathrm{~W}$ and $\tau=0.05 \mathrm{~s}$;

(d) $n_{\mathrm{e}}=10^{18} \mathrm{~m}^{-3}, P_{\text {LASER }}=300 \mathrm{~W}$ and $\tau=1 \mathrm{~s}$.

For each $n_{\mathrm{e}}$ and $P_{\text {LASER }}$ combination, $\tau$ is chosen to give relative errors less than $10 \%$ for a wide range of $T_{\mathrm{e}}$. From these figures we can say that: (a) a plasma with $n_{\mathrm{e}} \geq 10^{19} \mathrm{~m}^{-3}$ can be measured with time resolution of $0.1 \mathrm{~s}$; (b) a smaller laser power of $P_{\text {LASER }}=100 \mathrm{~W}$ results in a less favorable signal-to-noise ratio in $y_{i}$, which in turn requires an integration time longer than $0.5 \mathrm{~s}$ to yield reasonable data quality; (c) for a plasma ten times denser, $n_{\mathrm{e}}=10^{20} \mathrm{~m}^{-3}$, an integration time of $0.05 \mathrm{~s}$ is necessary for the same $P_{\text {LASER }}=300 \mathrm{~W}$; (d) a lower-density plasma of $n_{\mathrm{e}}=10^{18} \mathrm{~m}^{-3}$ can be measured with $P_{\text {LASER }}=300 \mathrm{~W}$ by a much longer integration time, up to $1 \mathrm{~s}$. We can see that $\sqrt{\left(\delta n_{\mathrm{e}}\right)^{2}} / n_{\mathrm{e}}<\sqrt{\left(\delta T_{\mathrm{e}}\right)^{2}} / T_{\mathrm{e}}$ for all the cases. This may be explained by the fact that $T_{\mathrm{e}}$ is deduced from the ratios among $\left\{y_{i}\right\}$, while $n_{\mathrm{e}}$ is deduced from $\left\{y_{i}\right\}$ directly. In practice, however, taking into account the error arising from a calibration process, the error in $n_{\mathrm{e}}$ is usually larger than the error in $T_{\mathrm{e}}$. The filter passband combination shown in Fig. 5 is not optimized to give the best performance. However, it is considered that the result shown in Fig. 9 will not change much with adjustment to the filter passband combination, but rather shows general features.

\section{Discussion}

We re-examined the feasibility of a fiber-based TSprobe 13 years after an initial proposal [1]. During this period, we observed great progress in high-power laser diodes, which may be used as a light source. A newly developed fiber feed-through is adopted in the design as a convenient optical component. It was shown that a currently available laser diode of $300 \mathrm{~W}$ can yield $10 \%$ accuracy in $T_{\mathrm{e}}$ and $n_{\mathrm{e}}$ data for a plasma of $n_{\mathrm{e}} \sim 10^{19} \mathrm{~m}^{-3}$ with an integration time of $0.1 \mathrm{~s}$. This may be useful for measuring slowly changing or steady-state plasma discharges.

Now we discuss possible ways of improving this performance, i.e., lowering errors and shortening the integra-

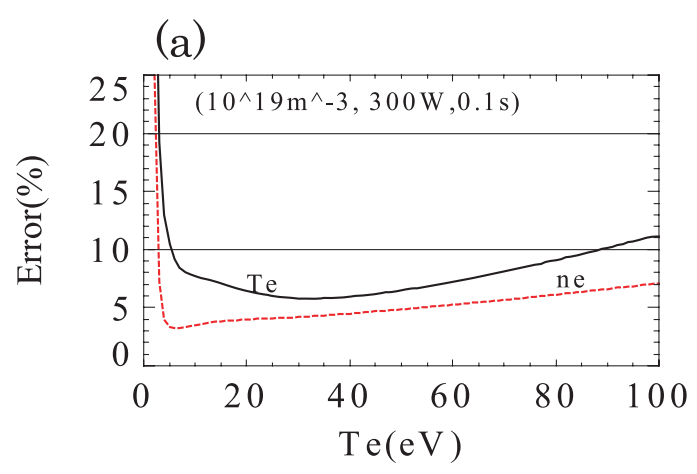

(b)

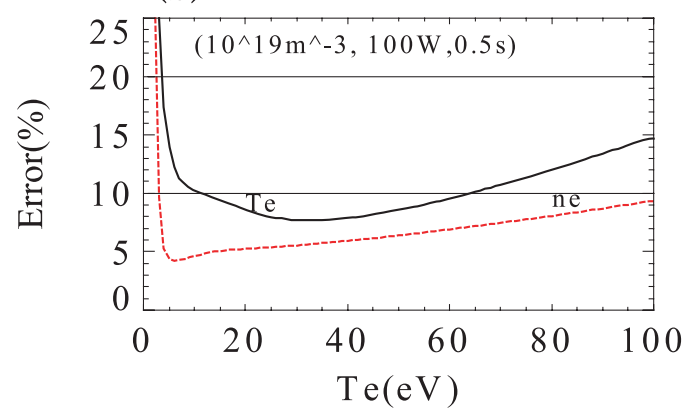

(c)

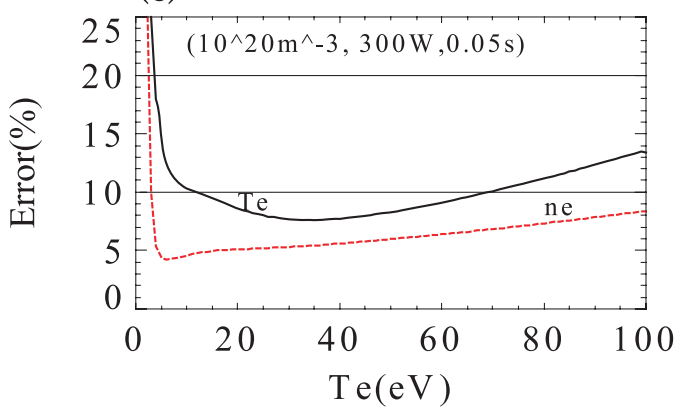

(d)

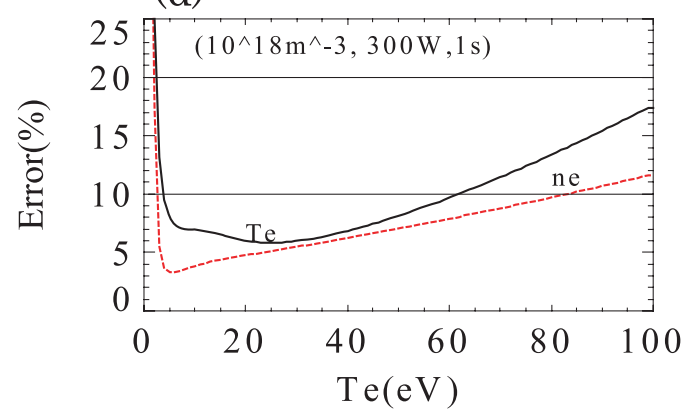

Fig. $9 \sqrt{\left(\delta T_{\mathrm{e}}\right)^{2}} / T_{\mathrm{e}}$ and $\sqrt{\left(\delta n_{\mathrm{e}}\right)^{2}} / n_{\mathrm{e}}$ for: (a) $n_{\mathrm{e}}=10^{19} \mathrm{~m}^{-3}$, $P_{\text {LASER }}=300 \mathrm{~W}$ and $\tau=0.1 \mathrm{~s}$; (b) $n_{\mathrm{e}}=10^{19} \mathrm{~m}^{-3}$, $P_{\text {LASER }}=100 \mathrm{~W}$, and $\tau=0.5 \mathrm{~s} ;$ (c) $n_{\mathrm{e}}=10^{20} \mathrm{~m}^{-3}$, $P_{\text {LASER }}=300 \mathrm{~W}$ and $\tau=0.05 \mathrm{~s}$; (d) $n_{\mathrm{e}}=10^{18} \mathrm{~m}^{-3}$, $P_{\mathrm{LASER}}=300 \mathrm{~W}$, and $\tau=1 \mathrm{~s}$.

tion time. In the above analyses, noise arises predominantly from the shot noise induced by plasma light enhanced by the avalanche process. An enhancement factor 
(a)

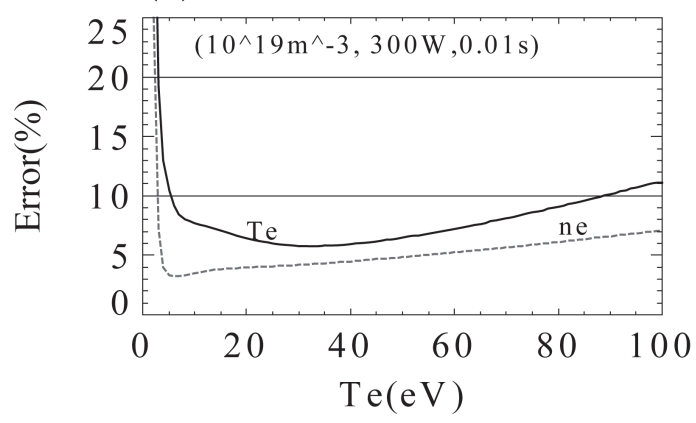

(b)

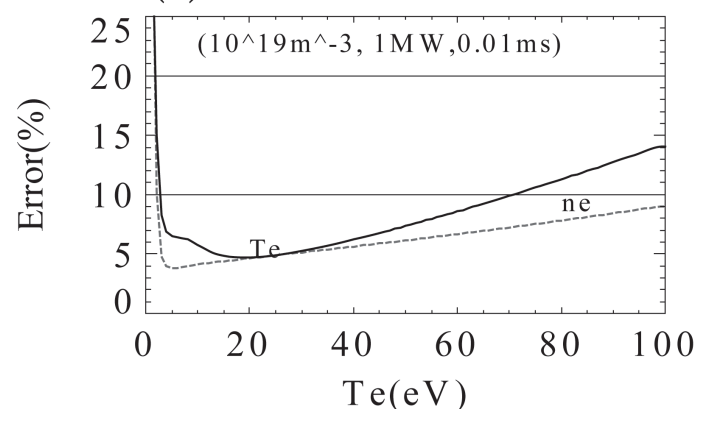

Fig. 10 (a) $\sqrt{\left(\delta T_{\mathrm{e}}\right)^{2}} / T_{\mathrm{e}}$ and $\sqrt{\left(\delta n_{\mathrm{e}}\right)^{2}} / n_{\mathrm{e}}$ for $P_{\mathrm{LASER}}=300 \mathrm{~W}$, $n_{\mathrm{e}}=10^{19} \mathrm{~m}^{-3}$, and $\tau=0.01 \mathrm{~s}$ without enhanced noise in the APD. (b) $\sqrt{\left(\delta T_{\mathrm{e}}\right)^{2}} / T_{\mathrm{e}}$ and $\sqrt{\left(\delta n_{\mathrm{e}}\right)^{2}} / n_{\mathrm{e}}$ for $P_{\mathrm{LASER}}=$ $1 \mathrm{MW}, n_{\mathrm{e}}=10^{19} \mathrm{~m}^{-3}$ and $\tau=0.01 \mathrm{~ms}$.

$F_{\mathrm{n}}(M)=10$ is supposed in this paper. If APD noise were unenhanced, that is, $F_{\mathrm{m}}(M)=1$, the noise power spectrum in Eq. (5) would be reduced appreciably. In this case, the errors in $T_{\mathrm{e}}$ and $n_{\mathrm{e}}$ are much reduced, and an integration time of $0.01 \mathrm{~s}$ is long enough to yield accurate data, as shown in Fig. 10 (a).

For this reason, developing less noisy photo detectors is desirable [10]. The second way of improving performance is to raise the probe light power. The key element of the TS-probe is intense light transmission in a fiber. A $\phi 1.2$ fiber, which is still flexible, can transmit CW light of $2 \mathrm{~kW}$ and $1 \mathrm{MW}$ pulses with duration less than $1 \mathrm{~ms}$. If a $1 \mathrm{MW}$ laser of $0.01 \mathrm{~ms}$ pulse width is available, it will provide enough accuracy with $\tau=0.01 \mathrm{~ms}$, as shown in Fig. 10 (b). The feasibility of such high-power light from a laser diode is beyond the scope of present paper. A solidstate laser such as a Nd: YAG laser pumped by a laser diode may yield such light, but it will be much less efficient, and hence, expensive. The third way of improving performance is to reduce the $V_{\mathrm{obs}}$ as much as possible. The assumed $V_{\text {obs }} \sim 10 \mathrm{~mm}^{3}$ is determined by the waist size $(\phi 1$ $\mathrm{mm}$ ) of the laser beam at the scattering position, which is largely dependent on the diameter and $N A$ of the lasertransmitting fiber. A smaller diameter and smaller $N A$ are favorable for reducing the beam size. If a laser diode powered by a flexible cable can be mounted on the TS-probe head, the beam size at the focal point may be reduced to $\phi 0.1$, thus lowering $V_{\text {obs }}$ and $P_{\text {rad }}$ by two orders of magnitude, if the beam quality is good enough.

We showed the feasibility of the TS-probe, but to realize it in practice, we must solve some technical issues, such as: (1) how to modulate $300 \mathrm{~W}$ laser output power at a high frequency of $\mathrm{MHz}$, (2) how to dump the high-power laser energy of $300 \mathrm{~W}$ without damaging the neighboring structures, and (3) how to lower stray light to a level at which the weak scattered light can be discriminated.

[1] K. Narihara et al., Fusion Eng. Des. 34-35, 67 (1997).

[2] Diaguide, Mitsubishi-cable Catalog 2000. 07 (in Japanese).

[3] http://www.lewvac.co.uk.

[4] http://www.LIMO.de.

[5] Laser Focus World Japan 8, 52 (2008).

[6] C. Moser, L. Ho, E. Maye and F. Havermeyer, J. Phys. D: Appl. Phys. 41, 224003 (2008).

[7] http://www.Thinkers.com/products/SR844.htm.

[8] A. van der Ziel, Noise, Prentice-Hall, Inc, 1954.

[9] R. J. McIntyre, IEEE Trans. Electron Devices ED-13, 164 (1966).

[10] 'The Avalanche Photodiode Catalog', Advanced Photonix, Inc. 